\title{
Trayectorias escolares universitarias de acuerdo con el capital cultural de los estudiantes de licenciatura de la UPSIN
}

\author{
University School Paths according to the Cultural \\ Capital of the UPSIN Undergraduate Students
}

\section{RESUMEN}

El propósito de este estudio radica en conocer la composición social de los estudiantes de la generación 2014-2018 de una Universidad Politécnica en México, así como la trayectoria escolar universitaria con que transitan los diferentes tipos de estudiantes de acuerdo con su Capital Cultural. En México, alrededor de $43.6 \%$ de la población tiene dificultades para acceder al bienestar económico, espacios de vivienda dignos, alimentación y servicios básicos; aunado a estas problemáticas, esa misma población también tiene que hacer frente a las deficiencias encontradas en las escuelas a las que asisten. La combinación de estas problemáticas invita a conocer qué sectores sociales llegan a la universidad y cuáles son sus logros escolares. Con el fin de alcanzar dicho propósito, este trabajo replicó el estudio de Araiza-Lozano y Aduelo (2017) basado en el estudio de Casillas et al. (2007) para su elaboración. Los resultados de este estudio hacen suponer que a los estudiantes con capitales familiares muy pobres no les fue posible reemplazar, con recursos propios, lo que la escuela no les proveyó para poder participar en estudios universitarios. Los estudiantes con capitales familiares muy pobres y con trayectorias escolares universitarias exitosas son prácticamente inexistentes.

Palabras clave: estudiantes universitarios, capital cultural, trayectorias escolares universitarias
Miguel Ángel Araiza Lozano*

\section{ABSTRACT}

The purpose of this study is to know the social composition of the students of the 2014-2018 generation of a Polytechnic University in Mexico, as well as the university trajectory with which the different types of students circulate according to their Cultural Capital. In Mexico, about $43.6 \%$ of the population has difficulties in accessing economic wellbeing, decent housing, food and basic services; coupled with these problems, the same population also has to face the deficiencies found in schools to which they attend. The combination of these problems invites us to know what social sectors come to the university and what their school achievements are. In order to achieve this purpose, this work replicated the study by Araiza-Lozano and Aduelo (2017) based on the study by Casillas et al. (2007) for its elaboration. The results of this study suggest that students with very poor family capitals were not able to replace, with their own resources, what the school did not provide them to participate in university studies. Students with very poor family capitals and successful university school pathways are practically nonexistent.

Key words: university students, cultural capital, university school trajectories

^Universidad Autónoma de Sinaloa, México; aralmia@gmail.com 


\section{INTRODUCCIÓN}

\section{Desigualdades desde el principio}

Con el fin de ayudar al lector a reflexionar en el esfuerzo titánico que han necesitado hacer algunos estudiantes universitarios para seguir dentro del sistema educativo -nos referimos a aquellos estudiantes que, proviniendo de contextos socioeconómicamente adversos, han logrado llegar a las aulas universitarias-, se ha decidido abordar brevemente el panorama económico y educativo en México de forma general.

La Organización para la Cooperación y el Desarrollo Económicos (OCDE) seńala que, entre los países que integran a esta organización, México tiene el mayor porcentaje de infantes en pobreza; además, sólo $10 \%$ de las familias posee $80 \%$ de los activos financieros. El coeficiente de Gini, indicador de la distribución de la riqueza, es de 0.459 , lo que hace a México el país con mayor desigualdad de ingresos entre los países miembros de esta organización (OCDE, 2018). Es así que México es un país de grandes desigualdades, con una población de casi 131 millones de habitantes, de los cuales $35.9 \%$ se encuentran en situación de pobreza y $7.6 \%$ en situación extrema, lo que significa que alrededor de $43.6 \%$ de la población tiene dificultades para gozar de bienestar económico, así como para acceder a la educación, servicios de salud, seguridad social, alimentación, espacios de vivienda dignos y servicios básicos en sus viviendas (CONEVAL, 2017b, 2017a; Countrymeters, 2017).

Asimismo, la Asociación Mexicana de Inteligencia de Mercado y Opinión (AMAI), agrupa y clasifica, según el índice de Niveles Socioeconómicos (NSE), a los hogares mexicanos en siete niveles (A/B, C+, C, C-, D+, D y E), los cuales reflejan la capacidad para satisfacer las necesidades de sus integrantes en materia de vivienda, salud, energía, tecnología, prevención y desarrollo intelectual. El nivel A/B es el segmento de la población con el nivel de vida más alto, representa 3.9\% de los hogares en México, el C+ es el siguiente nivel con mayor calidad de vida y lo compone $9.3 \%$, el nivel C lo constituye $10.7 \%$, el nivel C- $12.8 \%$, el nivel D+ 19\%, el nivel D, con el mayor porcentaje, está compuesto por 
$31.8 \%$, y el nivel E, que es el segmento con la menor calidad de vida, carece de todos los servicios y bienes satisfactores, representa $12.5 \%$ de la población (AMAI, 2016).

La problemática de la distribución de la riqueza en México no es la única desventaja que enfrentan los sectores sociales más desfavorecidos, sino que se les suman las deficiencias de las escuelas a las que asisten los estudiantes que pertenecen a estos sectores, pues la situación educativa de sus localidades y regiones también tienen deficiencias graves en su infraestructura, equipamiento, provisión de materiales, tecnología, formación docente, gestión escolar y programas de apoyo. Como consecuencia, también los resultados escolares de estos estudiantes son deficientes (Conafe, 2018; INEE, 2004, 2007; INEGI, 2016; OCDE, 2016). Frente a dichas deficiencias, según concluye Mejía (2013), a los estudiantes que provienen de familias en desventajas socioeconómicas no les es posible reemplazar, con recursos propios, lo que la escuela no les provee para poder participar, en un futuro próximo, en la construcción de una sociedad productiva y sustentable. Con puntualidad específica para los fines de este trabajo, nos referimos a las pocas probabilidades que tienen estos estudiantes de continuar con sus estudios universitarios.

\section{La investigación sobre los estudiantes universitarios y sus retos}

Dentro de las diversas declaraciones del gobierno federal sobre la educación, se retoman dos que se relacionan con el propósito de este trabajo, la primera afirma que es en la educación superior donde "cada estudiante debe lograr un sólido dominio de las disciplinas y valores que deben caracterizar a las distintas profesiones" (SEP, 2013, p. 27), y la segunda que "la población de menores ingresos en ocasiones tiene menos posibilidades de acceder a una educación de calidad y concluir sus estudios" (SEP, 2013, p. 29). Sin embargo, es imposible comprender la educación superior en México y los retos a los que sus estudiantes se enfrentan sin conocer quiénes son los alumnos que integran este sistema, de qué estratos sociales proceden y cómo es su desempeño académico.

No obstante, en México se considera reciente la profundización sobre el conocimiento de los estudiantes universitarios, pues 
su consolidación como campo de estudio ocurre a principios del siglo XXI. Cabe señalar que, al mismo tiempo, el sistema educativo empezó a reconocer en su discurso a los estudiantes universitarios, con políticas oficiales y programas institucionales orientados a mejorar la calidad educativa (ANUIES, 2000; SEP, 2001, 2007). De acuerdo con Guzmán (2011), las principales líneas de investigación en el campo son las siguientes:

- Ingreso y matrícula escolar.

- Vida estudiantil, experiencias e identidades.

- Jóvenes y nuevas figuras estudiantiles.

- Composición social de los estudiantes.

- Trayectorias escolares o educativas.

Entre los principales retos de investigación sobre los estudiantes universitarios, para la presente década, se plantean los siguientes: la necesidad de actualizar el conocimiento disponible y la profundización en las líneas de investigación existentes; así como la realización de estudios particulares en distintos contextos que favorezcan la construcción de una visión integral de los estudiantes mexicanos (Guzmán, 2011).

\section{Estructura social de los estudiantes universitarios}

Como ya se ha mencionado, un rasgo de las investigaciones sobre los estudiantes universitarios en la primera década del siglo XXI ha sido conocer su composición social, pues diversos trabajos han mostrado que no todos cuentan con condiciones favorables para continuar con sus estudios profesionales, ya que entre ellos existen diferencias socioculturales, lo que implica que hagan diferentes esfuerzos para continuar en la universidad (Casillas, de Garay, Vergara y Puebla, 2001). Al respecto, algunas investigaciones encontraron que los estudiantes que asisten a las universidades públicas generalmente provienen de familias de clase media y media baja; sin embargo, también a esas mismas universidades asisten estudiantes de las clases altas y bajas de la sociedad (Chain y Jácome, 2007; de Garay, 2002; Guzmán y Serrano, 2007). Asimismo, se encontró que los estudiantes de las universidades 
Tecnológicas provienen mayormente de sectores de la población con bajos recursos, y los estudiantes que asisten a universidades privadas provienen de sectores de la población con mayores recursos (de Garay, 2002).

De esta manera podemos observar una heterogeneidad de estudiantes universitarios, provenientes de los diferentes grupos sociales, algunos de los cuales, pese a provenir de diferentes estratos sociales, conviven en las mismas aulas de estudios. De esta forma gran parte de los estudiantes universitarios han cursado sus estudios profesionales con carencias, con apoyos institucionales como becas o descuentos en colegiaturas y combinando el trabajo con sus estudios (Guzmán, 2011; Miller, 2012). Como es de esperar, las carencias de las familias de estos universitarios también son culturales y escolares, por lo que un gran número de ellos ha tenido que vivir sus estudios universitarios con las desventajas inherentes de provenir de familias con escasos recursos socioeconómicos y culturales, además de una deficiente formación previa (Cu Balán, 2005; de Garay, 2005; Garbanzo, 2013; García-Castro y Bartolucci, 2007; Mejía, 2013).

\section{Capital cultural y trayectoria escolar}

A diferencia del capital económico, el capital cultual tiene la cualidad de no ser transmitido o heredado instantáneamente; por el contrario, es un proceso cimentado en diferentes elementos, personales y sociales. Según Bourdieu (1984), el capital cultural puede encontrarse en tres formas: en el estado incorporado, el individuo lo posee biológicamente y se termina o se desgasta a medida que el individuo se desgasta biológicamente; en el estado objetivado, es transmitido de forma tangible en bienes materiales como pinturas, libros, esculturas, etc., y, por último, en el estado institucionalizado, ejemplo de lo cual son los títulos escolares. Cabe mencionar que Bourdieu y Passeron (2009) denominaron de forma especial a aquellos estudiantes con más privilegios, de acuerdo con su capital cultural, para quienes se reserva, en varios sentidos, la mejor porción de la educación, a los cuales llamaron herederos. 
Ajustados a los términos de Bourdieu (1984), según Casillas, Chain y Jácome (2007), el capital cultural que poseen los estudiantes antes de entrar a la universidad, en su estado institucionalizado, es básicamente homogéneo, pues todos poseen los mismos diplomas (bachillerato aprobado y aceptación en los procesos de selección a la universidad). No obstante, para comprender el estado incorporado y objetivado, los autores recurren a diversas fuentes de información, de las que retoman algunas experiencias escolares anteriores y algunos rasgos socioeconómicos de la familia de la cual provienen los estudiantes.

De esta manera, con objeto de operacionalizar la noción de capital cultural, Casillas et al. (2007) han construido dos variables que se refieren a la noción de capital cultural de Bourdieu (1984), esto es, que, en su articulación y combinación específicas, son capaces de diferenciar a los estudiantes por su capital cultural conforme a dos propiedades principales: la influencia familiar y la influencia escolar. Con dicha operacionalización, Casillas et al. (2007) hacen evidente que existe una heterogeneidad de estudiantes. De acuerdo con sus resultados, estos autores afirman que la educación superior es excluyente, pues pone a competir a los estudiantes más necesitados con los más privilegiados en un mismo contexto educativo, y que en realidad la educación superior es un bien al cual accede sólo una minoría, reproduciendo así la desigualdad social.

En una investigación semejante, Araiza-Lozano y Audelo (2017) concluyen que existe una variedad evidente de estudiantes universitarios con distintas trayectorias escolares y capitales culturales, por lo que los alumnos y sus familias hacen diferentes esfuerzos para ingresar y egresar de la universidad. Esto debido a las desventajas socioeconómicas de los estudiantes menos favorecidos en comparación con sus pares, que provienen de familias con mejores recursos socioeconómicos y culturales.

Es así que siguiendo el patrón recomendado por Guzmán (2011) para actualizar el conocimiento sobre quiénes son los estudiantes universitarios, este trabajo, el cual es una réplica del estudio de Araiza-Lozano y Audelo (2017) basados en el estudio de Casillas et al. (2007) para su elaboración, tiene el propósito de conocer la composición social de los estudiantes de la generación 
2014-2018 de la Universidad Politécnica de Sinaloa (UPSIN) y la trayectoria escolar universitaria de los diferentes tipos de estudiantes de acuerdo con su Capital Cultural, planteando las siguientes preguntas a los estudiantes de la generación mencionada ¿Cómo son sus trayectorias escolares universitarias? ¿Qué dotaciones de Capital Cultural tienen? ¿Cómo son sus trayectorias escolares universitarias de acuerdo con su Capital Cultural?

\section{METODOLOGíA E INSTRUMENTO}

\section{Trayectorias Escolares Universitarias}

En este estudio, las Trayectorias Escolares Universitarias (TEU) son comprendidas como las formas académicas en que los estudiantes transitan por la universidad, clasificadas en cuatro niveles: Altas, Regulares, Bajas y Muy bajas (ver cuadro 1). Para hacer esta clasificación, se utilizaron tres aspectos básicos de la vida académica del estudiante: el Promedio de Calificaciones (PC), el Índice de Aprobación en Ordinario (ÍAO) y el Índice de Promoción de sus Cursos (IIPC; Casillas et al., 2007; Chaín, 1994).

\section{CUADRO 1.}

\begin{tabular}{|l|c|}
\hline \multicolumn{1}{|c|}{ Clasificación TEU } & Puntos \\
\hline Alta & 100 a 90 \\
\hline Regular & 89 a 80 \\
\hline Baja & 79 a 70 \\
\hline Muy baja & 69 y menos \\
\hline
\end{tabular}

Fuente: Araiza-Lozano y Audelo (2017).

Para el PC obtenido por los estudiantes, se tomó en cuenta hasta el último cuatrimestre que tenían concluido al momento del estudio. El ÍAO clasificó a los estudiantes según el grado de aprobación en evaluaciones ordinarias; el cálculo se estableció poniendo en relación las materias cursadas con las materias recursadas. El ÍPC se estableció poniendo en relación el número total de 
materias promovidas, entre el total de materias que los estudiantes debieron haber acreditado de acuerdo con el plan de estudios de su carrera (Casillas et al., 2007; Chaín, 1994). Con base en lo anterior, se desarrolló la fórmula para ponderar el índice TEU, la cual se muestra a continuación.

$$
\mathrm{TEU}=[(\mathrm{PC})+(\mathrm{I} A 0-100)+(\mathrm{I} \mathrm{PC}-100)]
$$

\section{Capital Cultural}

El Capital Cultural (CC) es el conjunto de hábitos de vida y de trabajo; de formas de ser y de comportarse; de conocimientos y habilidades desarrolladas; de acceso a recursos culturales, sociales y materiales. Dichos recursos se obtienen a través de largos procesos de socialización, desarrollados principalmente en el contexto familiar, escolar y social. Por lo que la noción de CC hace observable la historia social del individuo (Casillas et al., 2007).

Los bienes culturales están mediados por el origen social de las familias de los estudiantes, y por su propio esfuerzo e interés para conseguirlos. Si bien el factor económico suele ser relevante, no es lo único para favorecer dicha apropiación. Es por ello que la noción de CC diferencia a los estudiantes de acuerdo con sus disposiciones culturales y no sólo por sus posesiones económicas (Bourdieu, 2005). Es así que el CC está conformado por los índices: Capital Familiar y Capital Escolar (Casillas et al., 2007).

\section{Capital Cultural = Capital Familiar + Capital Escolar}

Si bien ambos índices están relacionados, en la trayectoria escolar es observable el trabajo del estudiante, la inversión y valorización que éste tiene por sus estudios. Básicamente en estos dos índices, Capital Familiar y Capital Escolar, se hacen indiscutibles las capacidades de la familia para llevar al estudiante a cierto nivel educativo, así como los recursos propios del estudiante para desempeñarse en su vida escolar. 


\section{Capital Familiar}

Este índice incluye a los padres de los estudiantes, su escolaridad y el nivel de competencia de su ocupación, además del nivel socioeconómico de la familia. En este índice se reúne un conjunto de disposiciones sociales, en las que se incluyen atributos y recursos que poseen los estudiantes de acuerdo con el origen social que les brinda su familia (Casillas et al., 2007). De tal manera que:

Capital Familiar $=$ Escolaridad de los padres + Nivel de competencias de la ocupación de los padres + Nivel socioeconómico de la familia

Para clasificar la escolaridad de los padres de los estudiantes se tomó como base la Estructura del Sistema Educativo Nacional de México. La Ley General de Educación establece tres tipos de educación: básica, media superior y superior (Ordorika y Rodríguez, 2012). La clasificación escolar en la que se ubica a los padres va de ninguna a posgrado: menos de primaria completa, primaria o secundaria, preparatoria o carrera técnica, carrera universitaria y posgrado. Para clasificar el nivel de competencias de la ocupación de los padres, se utilizó el "Sistema nacional de clasificación de ocupaciones, 2011, SINCO” (INEGI, 2011), el cual considera uno o más de los siguientes elementos: la naturaleza del trabajo; el nivel de enseñanza formal y la cantidad de formación informal o la experiencia previa.

Con base en estos tres indicadores, el nivel de competencia de la ocupación de los padres se agrupó en nueve niveles de especialización, comenzando con las tareas de más alta especialización hasta las de más baja: Funcionarios, directores y jefes; profesionistas y técnicos; trabajadores auxiliares en actividades administrativas; comerciantes, empleados en ventas y agentes de ventas; trabajadores en servicios personales y vigilancia; trabajadores en actividades agrícolas, ganaderas, forestales, caza y pesca; trabajadores artesanales; operadores de maquinaria industrial, ensambladores, choferes y conductores de transporte; trabajadores en actividades elementales y de apoyo. 
El Índice de Nivel Socioeconómico (NSE), creado por la Asociación Mexicana de Inteligencia de Mercado y Opinión (AMAI) se utilizó para calcular el NSE de las familias de los estudiantes. Dicho índice permite clasificar a los hogares mexicanos en siete niveles (ver cuadro 2), según su capacidad para satisfacer las necesidades más importantes de sus integrantes: salud, prevención, energía, tecnología, vivienda y desarrollo intelectual. El grado de satisfacción de dichas necesidades establece el nivel de calidad de vida y bienestar de los integrantes del hogar (AMAI, 2016).

CUADRO 2. Nivel Socioeconómico

\begin{tabular}{|l|l|c|}
\hline \multicolumn{1}{|c|}{ Nivel } & \multicolumn{1}{|c|}{ Principal característica } & Distribución de los hogares por NSE en México \\
\hline AB & Planeación y futuro & $6.8 \%$ \\
\hline C+ & Entretenimiento y comunicación & $14.2 \%$ \\
\hline C & Vida práctica & $17.0 \%$ \\
\hline C- & Mínimo de practicidad & $17.1 \%$ \\
\hline D+ & Condiciones básicas sanitarias & $18.5 \%$ \\
\hline D & Paredes y algunos servicios & $21.4 \%$ \\
\hline E & Escasez & $5.0 \%$ \\
\hline
\end{tabular}

Fuente: AMAI (2016).

\section{Clasificación de Capital Familiar}

Se hizo una clasificación del índice CF fijando puntos para cada categoría por orden jerárquico. Los puntos logrados en cada elemento se sumaron para clasificar a cada estudiante en una categoría. La clasificación va de CF Alto a Muy Pobre (ver cuadros 3 y 4).

\section{Capital Escolar}

El Capital Escolar (CE) es el segundo índice que conforma el CC. $\mathrm{El} \mathrm{CE}$ es el conjunto de atributos académicos de los estudiantes, resultado de un largo proceso de socialización escolar. Para este estudio se tomó en cuenta la trayectoria escolar inmediata anterior a la educación superior, a través de los siguientes indicadores: 
TRAYECTORIAS ESCOLARES UNIVERSITARIAS DE ACUERDO CON EL CAPITAL CULTURAL ...

CUADRO 3. Puntuación asignada a cada elemento del Capital Familiar

\begin{tabular}{|c|c|c|c|c|c|}
\hline Escolaridad de los padres & Puntos & Índice socioeconómico & Puntos & Ocupación de los padres & Puntos \\
\hline Posgrado & 4 & Nivel A/B & 7 & $\begin{array}{l}\text { 1. Funcionarios, directores } \\
\text { y jefes }\end{array}$ & 9 \\
\hline Carrera universitaria & 3 & Nivel C+ & 6 & 2. Profesionistas y técnicos & 8 \\
\hline $\begin{array}{l}\text { Preparatoria o carrera } \\
\text { técnica }\end{array}$ & 2 & Nivel C & 5 & $\begin{array}{l}\text { 3. Trabajadores auxiliares en } \\
\text { actividades administrativas }\end{array}$ & 7 \\
\hline Primaria o secundaria & 1 & Nivel C- & 4 & $\begin{array}{l}\text { 4. Comerciantes, empleados } \\
\text { en ventas y agentes de } \\
\text { ventas }\end{array}$ & 6 \\
\hline \multirow[t]{5}{*}{$\begin{array}{l}\text { Menos de primaria } \\
\text { completa }\end{array}$} & 0 & Nivel D+ & 3 & $\begin{array}{l}\text { 5. Trabajadores en servicios } \\
\text { personales y vigilancia }\end{array}$ & 5 \\
\hline & & Nivel D & 2 & $\begin{array}{l}\text { 6. Trabajadores en actividades } \\
\text { agrícolas, ganaderas, } \\
\text { forestales, caza y pesca }\end{array}$ & 4 \\
\hline & & Nivel E & 1 & 7. Trabajadores artesanales & 3 \\
\hline & & & & $\begin{array}{l}\text { 8. Operadores de maquinaria } \\
\text { industrial, ensambladores, } \\
\text { choferes y conductores de } \\
\text { transporte }\end{array}$ & 2 \\
\hline & & & & $\begin{array}{l}\text { 9. Trabajadores en actividades } \\
\text { elementales y de apoyo }\end{array}$ & 1 \\
\hline
\end{tabular}

Fuente: Araiza-Lozano y Audelo (2017), basados en Casillas et al. (2007).

CUADRO 4. Clasificación de Capital Familiar

\begin{tabular}{|l|c|c|c|c|c|}
\hline \multicolumn{1}{|c|}{ Clasificación } & \multicolumn{5}{c|}{ Puntos } \\
\hline Alto & & 17 & 18 & 19 & 20 \\
\hline Medio & 12 & 13 & 14 & 15 & 16 \\
\hline Pobre & 7 & 8 & 9 & 10 & 11 \\
\hline Muy pobre & 2 & 3 & 4 & 5 & 6 \\
\hline
\end{tabular}

Fuente: Araiza-Lozano y Audelo (2017).

Capital Escolar=Promedio de bachillerato + Reprobador o no en el bachillerato + Año de egreso del bachillerato-Ingreso a la universidad + Calificación obtenida en el examen de ingreso a la universidad 
Se entiende el promedio de bachillerato como la unidad de medida utilizada para observar el desempeño académico de los estudiantes; sintetiza, en una nota, la diversidad de calificaciones obtenidas por los alumnos en este nivel educativo, donde de cero a cinco se consideran calificaciones reprobatorias y de seis a 10 calificaciones aprobatorias. El indicador, estudiante reprobador o no en el bachillerato, muestra si el estudiante tuvo en sus estudios de bachillerato dificultades académicas o si tuvo una trayectoria escolar académicamente estable. En este estudio se considerará que un estudiante es reprobador en el bachillerato si reprobó una o más materias. El año de egreso del bachillerato e ingreso a los estudios universitarios representa una trayectoria escolar continua o discontinua entre el bachillerato y el ingreso a la licenciatura, indica si el estudiante ingresó a la universidad el mismo año que egresó del bachillerato o lo hizo después.

Por otra parte, para hacer la selección de los futuros universitarios, diversas universidades en México, utilizan el EXANI-II como prueba de admisión; este examen proporciona información integral sobre los aspirantes que, según el Centro Nacional de Evaluación para la Educación Superior (CENEVAL), cuentan con mayores posibilidades de éxito para cursar estudios universitarios, así como conocer su nivel de desempeño en áreas esenciales para el comienzo de estudios universitarios (CENEVAL, 2018). El EXANI-II cuenta con 112 reactivos, 100 para calificar, 10 a prueba y dos de control. Las calificaciones del examen se muestran en una escala nombrada como Índice CENEVAL (ICNE), las cuales van de 700 a 1300 puntos; obtener 700 puntos equivale a la calificación más baja y obtener 1300 equivale a la calificación más alta; la media técnica es de 1000 puntos y representa $50 \%$ de aciertos (CENEVAL, 2018).

\section{Clasificación de Capital Escolar}

A continuación, se muestra cómo se hizo la clasificación del índice de CE, las puntuaciones asignadas a cada elemento del CE y su clasificación a través de la asignación de puntos para cada categoría, los cuales están asignados por orden jerárquico. Es así que los puntos alcanzados por cada estudiante en cada elemento 
se suman para clasificarse en una categoría. La clasificación de CE va de Alto a Muy bajo (ver cuadros 5 y 6 ).

CUADRO 5. Puntuación asignada a cada elemento del Capital Escolar

\begin{tabular}{|c|c|c|}
\hline \multicolumn{1}{|c|}{ Promedio prepa } & Clasificación & Puntos asignados \\
\hline $10-9$ & Alto & 3 \\
\hline $8.9-8$ & Medio & 2 \\
\hline $7.9-7$ & Bajo & 1 \\
\hline Reprobador en bachillerato & Clasificación & Puntos asignados \\
\hline 0 materias reprobadas & No reprobador & 1 \\
\hline 1 y más materias reprobadas & Reprobador & 0 \\
\hline Año del egreso y el año de ingreso a la universidad. & Clasificación & Puntos asignados \\
\hline Mismo año & Alto & 3 \\
\hline 2 años & Medio & 2 \\
\hline 3 años y más & Bajo & 1 \\
\hline Calificación obtenida en el examen de admisión & Clasificación & Puntos asignados \\
\hline $96 \%$ a $70 \%$ & Alto & 3 \\
\hline $69 \%$ a $56 \%$ & Medio & 2 \\
\hline $55 \%$ a 23\% & Bajo & 1 \\
\hline
\end{tabular}

Fuente: Araiza-Lozano y Audelo (2017), basado en Casillas et al. (2007).

\section{CUADRO 6. Clasificación de Capital Escolar}

\begin{tabular}{|l|c|c|}
\hline \multicolumn{1}{|c|}{ Clasificación } & \multicolumn{2}{c|}{ Puntos } \\
\hline Alto & 9 & 10 \\
\hline Medio & 7 & 8 \\
\hline Bajo & 5 & 6 \\
\hline Muy bajo & 3 & 4 \\
\hline
\end{tabular}

Fuente: Araiza-Lozano y Audelo (2017)).

\section{Instrumento}

Para conocer el NSE de los estudiantes se utilizó el cuestionario "Regla AMAI 8X7", creado por la Asociación Mexicana de Agencias de Investigación de Mercado (AMAI), el cual es un algoritmo 
que mide la satisfacción de las necesidades más importantes en el hogar del que provienen los estudiantes: vivienda, salud, energía, tecnología, prevención y desarrollo intelectual. El algoritmo "Regla AMAI 8X7" está constituido por ocho preguntas, las que consideran ocho características o posesiones del hogar y la escolaridad de la persona que más aporta al gasto en el hogar (AMAI, 2016).

Los niveles socioeconómicos, empezando por el más alto al más bajo son: A/B, C+, C, C-, D+, D y E. La aplicación del cuestionario SNE $8 \times 7$ se hizo en las aulas de la universidad, previa autorización de las autoridades escolares y la aprobación de los estudiantes. El instrumento fue previamente pilotado con el fin de generar familiaridad con su uso. Como preguntas adicionales, en el mismo instrumento, clasificaron la ocupación de sus padres de acuerdo con el "Sistema nacional de clasificación de ocupaciones 2011 SINCO”, además de mencionar su ańo de egreso del bachillerato e ingreso a la licenciatura (INEGI, 2011).

\section{Procedimientos de recolección y organización de la información}

Previa autorización de las autoridades correspondientes de la universidad, se gestionó la base de datos a través del Departamento de Control Escolar, de la cual se obtuvo información sobre: el promedio de preparatoria de los estudiantes, las calificaciones obtenidas en el examen de admisión para la universidad, la cantidad de materias reprobadas en el bachillerato, el promedio de calificaciones hasta el último cuatrimestre cursado en el momento del estudio, la cantidad de materias cursadas, aprobadas y reprobadas, así como la cantidad de materias recursadas y la escolaridad de los padres. El tratamiento estadístico de los datos obtenidos se llevó a cabo en el programa Statistical Package for the Social Sciences (SPSS; IBM, 2011).

\section{Sujetos Participantes}

La población objetivo, estuvo conformada por los estudiantes de la generación 2014-2018 de la UPSIN, los cuales, al momento 
de la realización de este estudio, cursaban el $8^{\circ}$ cuatrimestre de sus carreras universitarias, siendo los estudiantes más próximos a egresar. Los participantes pertenecen a las ingenierías presenciales: Biomédica, en Animación y Efectos Visuales, en Biotecnología, en Energía, en Informática, en Logística y Transporte, en Nanotecnología, en Tecnología Ambiental, Mecatrónica y la licenciatura en Terapia Física.

Pese a que se buscó trabajar con toda la población antes mencionada, no fue posible aplicar el instrumento de nivel socioeconómico a todos los estudiantes; varios de ellos, por diversas razones, no asistieron a sus clases regulares el día de la aplicación. Los estudiantes que no contestaron dicha encuesta no pudieron participar en el estudio, lo mismo que los estudiantes que no formaban parte de la generación 2014-2018; nos referimos a los que, por cualquier razón, se encontraban rezagados de su propia generación.

Tampoco pudieron participar los estudiantes que decidieron permanecer anónimos en la encuesta de nivel socioeconómico y los que no la contestaron completamente. De tal manera que en lista existían 634 estudiantes; sin embargo, de ese listado, la universidad no tenía los registros completos de 136 estudiantes, y dentro del mismo listado se encontraron 42 estudiantes rezagados, por lo que sólo 456 estudiantes eran elegibles para participar en el estudio. Al final, la cantidad de participantes fue de 420 estudiantes. Las bases de datos para obtener el índice de TEU corresponden a la conclusión del $7^{\circ}$ cuatrimestre.

\section{RESULTADOS}

\section{Trayectorias Escolares Universitarias}

Con base en los tres indicadores (PC, ÍAO e ÍPC) de las TEU se obtuvo la siguiente información. El índice TEU más elevado que se encontró fue de 99.3, mientras que el índice más bajo fue de 52.7, la mediana se ubicó en el valor 88.7, la media es igual a 86.7 y la moda es de 90 . Por otra parte, los valores que más se repitieron están dentro del rango 89.7-93.4 (ver figura 1). 
FIGURA 1. Histograma TEU

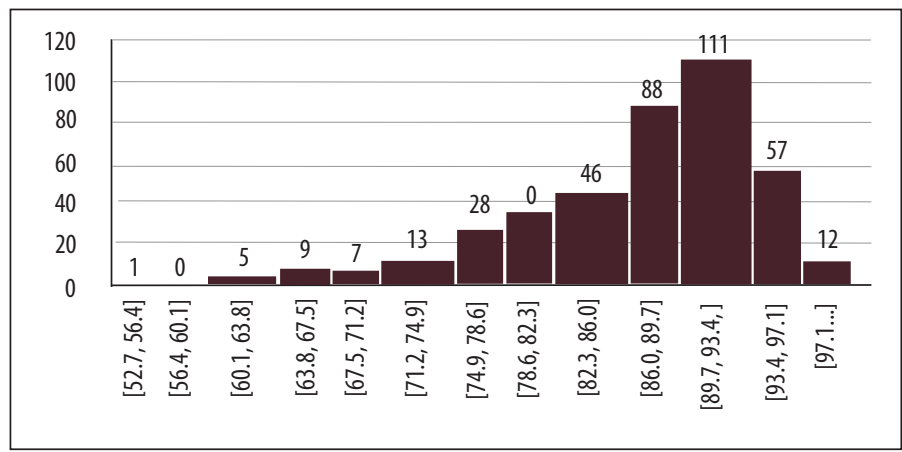

Fuente: elaboración propia.

A continuación, se muestran los tipos de Trayectorias Escolares Universitarias según los resultados del índice TEU. Alrededor de cuatro de cada 10 estudiantes presentaron trayectorias escolares Altas. Un resultado similar se observó en las trayectorias escolares denominadas como Regulares, ya que cuatro de cada 10 estudiantes presentan este tipo de trayectorias, alrededor de uno de cada 10 de estos estudiantes transitó por la universidad con una trayectoria escolar Baja, y casi uno de cada 20 con una trayectoria escolar Muy baja (ver cuadro 7).

CUADRO 7. Tipo de Trayectorias Escolares Universitarias

\begin{tabular}{|l|c|c|c|c|c|}
\hline Núm. /\% & Muy bajas & Bajas & Regulares & Altas & TOTAL \\
\hline Núm. & 20 & 55 & 165 & 173 & 413 \\
\hline$\%$ & 4.8 & 13.3 & 40.0 & 41.9 & 100.0 \\
\hline
\end{tabular}

Fuente: elaboración propia, basado en Casillas et al. (2007).

\section{Capital Familiar}

Los resultados relacionados con el CF mostraron que alrededor de cuatro de cada 10 estudiantes universitarios se clasificaron con un CF Medio, y alrededor de tres de cada 10 con un CF Alto, por 
lo que alrededor de $70 \%$ de la población estudiada se clasificaron como estudiantes con CF Alto y Medio, mientras que 30\% presentó un CF Pobre y Muy pobre; sin embargo, se destaca que alrededor de tres de cada 100 estudiantes se clasificaron como Muy pobres (ver cuadro 8).

CUADR0 8. Clasificación de CF

\begin{tabular}{|l|c|c|}
\hline \multicolumn{1}{|c|}{ Clasificación } & Núm. & $\%$ \\
\hline Alto & 118 & 28.6 \\
\hline Medio & 172 & 41.6 \\
\hline Pobre & 109 & 26.4 \\
\hline Muy pobre & 14 & 3.4 \\
\hline TOTAL & 413 & 100 \\
\hline
\end{tabular}

Fuente: elaboración propia, basado en Casillas et al. (2007).

\section{Capital Escolar}

Los resultados referentes al CE mostraron que alrededor de cuatro de cada 10 estudiantes universitarios se clasificaron con un CE Alto, y alrededor de cuatro de cada 10 con un CE Medio, por lo que alrededor del $80 \%$ de la población estudiada se clasificó como estudiantes con CE Alto y Medio, mientras que alrededor de 20\% se ubicó en la población clasificada con CE Bajo y Muy bajo (ver cuadro 9).

\section{Caracterización de los estudiantes por Capital Cultural}

La combinación del CF y del CE dio como resultado la siguiente clasificación (ver cuadro 10). Haciendo alusión al tipo de estudiantes que Bourdieu y Passeron (2009) denominaron herederos, los estudiantes con un CF favorable, alto o medio, y un CE favorable, alto o medio, fueron denominados Herederos; a los estudiantes con un capital favorable y uno desfavorable se les denominó en Riesgo, ya que en algún sentido escolar o social pueden ser vulnerables. No obstante, a los estudiantes con los dos capitales desfavorables se les denominó en Alto riesgo, pues la precariedad de su 
estado escolar y social hace que su vulnerabilidad aumente (Arzate y González, 2007; Casillas et al., 2007; Guzmán, 2004, 2012; Guzmán y Saucedo, 2005; Hernández, 2016; Pérez-Sánchez y Castejón, 2009; Rodríguez et al., 2013; Ruiz, 2012; Silva, 2011).

\section{CUADRO 9. Clasificación de CE}

\begin{tabular}{|l|c|c|}
\hline \multicolumn{1}{|c|}{ Clasificación } & Núm. & $\%$ \\
\hline Alto & 161 & 38.3 \\
Medio & 183 & 43.6 \\
Bajo & 67 & 16.0 \\
Muy bajo & 9 & 2.1 \\
TOTAL & 420 & 100 \\
\hline
\end{tabular}

Fuente: elaboración propia, basado en Casillas et al. (2007).

Sin embargo, se encontraron algunos estudiantes que presentaron CE muy favorables a pesar de provenir de un contexto desfavorable, CF pobre o muy pobre; este tipo de estudiantes fueron denominados como Héroes y Superhéroes. El nombre que se les dio a estos estudiantes buscó hacer evidente el esfuerzo que debieron hacer para lograr entrar y permanecer en la universidad con buenas notas, pese a su contexto precario. Estos estudiantes de alguna manera lograron superar los obstáculos que se encuentran en su medio (Blanco, 2010; Camposeco, 2012; Gómez y Kotliarenco, 2010; Oros, 2009; Silas, 2008).

\section{Capital Cultural}

A continuación, se muestran los resultados relacionados con el CC, compuesto por el CF y el CE. Se puede observar en los resultados sobre este índice, que alrededor de seis de cada 10 de los estudiantes que participaron en esta investigación se clasificaron dentro del grupo de los Herederos, alrededor de cinco de cada 20 se clasificaron como en Riesgo, y cerca de dos de cada 30 se clasificaron como en Alto riesgo, alrededor de cinco de cada 60 
estudiantes se clasificaron como Héroes y se pudieron encontrar cinco Superhéroes por cada 413 estudiantes (ver cuadro 11).

CUADRO 10. Caracterización por Capital Cultural

\begin{tabular}{|l|c|c|}
\hline Capital Familiar & Capital Escolar & Tipo de alumno \\
\hline \multirow{4}{*}{ Muy Pobre } & Alto & Superhéroes \\
\cline { 2 - 3 } & Medio & Riesgo \\
\cline { 2 - 3 } & Bajo & Alto riesgo \\
\hline \multirow{4}{*}{ Pobre } & Alto & Héroes \\
\hline \multirow{4}{*}{ Alto } & Medio & Riesgo \\
\cline { 2 - 3 } & Bajo/Muy bajo & Alto riesgo \\
\hline \multirow{4}{*}{ Medio } & Alto & Herederos \\
\cline { 2 - 3 } & Medio & Herederos \\
\cline { 2 - 3 } & Bajo & Riesgo \\
\hline & Alto & Herederos \\
\cline { 2 - 3 } & Medio & Herederos \\
\cline { 2 - 3 } & Bajo & Riesgo \\
\hline & Muy bajo* & Alto riesgo \\
\hline
\end{tabular}

Fuente: elaboración propia, basado en Casillas et al. (2007).

* Sólo los grupos de estudiantes denominados como capital familiar medio y pobre presentaron capitales escolares muy bajos, por lo que la nomenclatura de capital escolar muy bajo únicamente aparece en dichos grupos de estudiantes.

\section{CUADR0 11. Clasificación por Capital Cultural}

\begin{tabular}{|l|c|c|}
\hline \multicolumn{1}{|c|}{ Tipo de estudiantes } & No & $\%$ \\
\hline Herederos & 240 & 58.1 \\
\hline Riesgo & 107 & 25.9 \\
\hline Alto riesgo & 27 & 6.5 \\
\hline Superhéroes & 5 & 1.2 \\
\hline Héroes & 34 & 8.2 \\
\hline Total & 413 & 100 \\
\hline
\end{tabular}

Fuente: elaboración propia, basado en Casillas et al. (2007). 


\section{Trayectorias Escolares Universitarias por grupos de Capital Cultural}

A continuación, se muestran las trayectorias escolares universitarias por grupos de CC. En primera instancia, en relación con el total de la población estudiada, el grupo más numeroso, Herederos, presentó también la mayor cantidad de trayectorias escolares Altas, alrededor de tres de cada 10; seguido del grupo identificado como en Riesgo, casi uno de cada 12; el grupo de estudiantes denominados como Héroes, con alrededor de uno de cada 25; después los estudiantes denominados como en Alto riesgo, uno de cada 100, y sólo uno de cada 400 de los estudiantes denominados como Superhéroes (ver cuadro 12).

En cuanto las trayectorias escolares universitarias denominadas como Regulares, alrededor de ocho de cada 35 de los estudiantes denominados como Herederos presentó este tipo de trayectoria; los estudiantes en Riesgo que presentaron esta trayectoria, fueron alrededor de uno de cada 10; de los estudiantes denominados como en Alto riesgo, alrededor de uno de cada 33; los considerados en el grupo de los Héroes, alrededor de uno de cada 30 y de los denominados como Superhéroes alrededor de tres de cada 400 (ver cuadro 12).

En cuanto a las trayectorias escolares denominadas como Bajas, alrededor de uno de cada 20 de los estudiantes denominados como Herederos presentaron este tipo de trayectoria; casi uno de cada 20 de los estudiantes denominados como Riesgo presentó este tipo de trayectoria; alrededor de uno de cada 100 del grupo denominado como Héroes; uno de cada 400 de los denominados como Superhéroes y tres de cada 200 de los denominados como en Alto riesgo (ver cuadro 12).

Por último, las trayectorias escolares denominadas como Muy bajas, las integraron los grupos de estudiantes denominados como Herederos, casi uno de cada 40; en Riesgo, tres de cada 200, y en Alto riesgo, uno de cada 100 (ver cuadro 12). 
TRAYECTORIAS ESCOLARES UNIVERSITARIAS DE ACUERDO CON EL CAPITAL CULTURAL ...

CUADRO 12. Trayectorias estudiantiles por grupos de capital cultural

\begin{tabular}{|l|c|c|c|c|c|c|c|c|c|c|}
\hline \multirow{2}{*}{ Tipo de Estudiante } & \multicolumn{8}{|c|}{ Tipo de Trayectoria Escolar Universitaria } & \multicolumn{3}{|c|}{} \\
\cline { 2 - 14 } & \multicolumn{2}{|c|}{ Alta } & \multicolumn{2}{|c|}{ Regular } & \multicolumn{2}{|c|}{ Baja } & \multicolumn{2}{|c|}{ M. Baja } & \multicolumn{3}{|c|}{ Total } \\
\cline { 2 - 14 }$y$ & $\%$ & No. & $\%$ & No & $\%$ & No & $\%$ & No & $\%$ & No \\
\hline Superhéroes & 0.2 & 1 & 0.7 & 3 & 0.2 & 1 & 0.0 & 0 & 1.2 & 5 \\
\hline Héroes & 3.9 & 16 & 3.4 & 14 & 1.0 & 4 & 0.0 & 0 & 8.2 & 34 \\
\hline Herederos & 28.3 & 117 & 22.0 & 91 & 5.3 & 22 & 2.4 & 10 & 58.1 & 240 \\
\hline Riesgo & 8.5 & 35 & 10.7 & 44 & 5.3 & 22 & 1.5 & 6 & 25.9 & 107 \\
\hline Alto riesgo & 1.0 & 4 & 3.1 & 13 & 1.5 & 6 & 1.0 & 4 & 6.5 & 27 \\
\hline Total & 41.9 & 173 & 40.0 & 165 & 13.3 & 55 & 4.8 & 20 & 100 & 413 \\
\hline
\end{tabular}

Fuente: elaboración propia, basado en Casillas et al. (2007).

\section{DISCUSIÓN Y CONCLUSIONES}

De conformidad con el propósito de esta investigación a continuación se trata la composición social de los estudiantes de la generación 2014-2018 de la Universidad Politécnica de Sinaloa, así como las Trayectorias Escolares Universitarias de dicha generación, según su Capital Cultural (CC).

Como ya se ha mencionado, una gran cantidad de estudiantes en México se enfrentan a desigualdades económicas (OCDE, 2018) y educativas (Mejía, 2013) desde el principio de sus estudios. Se puede considerar que los estudiantes que participaron en esta investigación son un reflejo de esas características, tal parece que los estudiantes con muy bajos recursos (sociales, económicos y culturales), tienen pocas posibilidades de asistir a la universidad pública donde se llevó acabo esta investigación, ya que alrededor de $70 \%$ de la población está clasificada con CF Altos y Medios. En otras palabras, estos estudiantes pertenecen a los niveles socioeconómicos más favorecidos de la población, con padres que generalmente cuentan con estudios superiores y ocupaciones con altos niveles de competencia. Únicamente 3.4\% de la población participante se clasifica con un CF Muy pobre. 
Estos hallazgos no deben sorprender, ya que los datos del CONEVAL (2017a, 2017b), indican que más de 43\% de la población nacional tiene dificultades para acceder al bienestar económico, así como a servicios básicos en sus viviendas, servicios de salud, seguridad social, alimentación, espacios de vivienda dignos y acceso a la educación. Además, como ya se ha mencionado, los estudiantes con un CF Muy pobre, suelen vivir deficiencias escolares relacionadas con infraestructura, equipamiento, provisión de materiales y tecnología, formación docente, gestión escolar y programas de apoyo (Conafe, 2018; INEE, 2004, 2007; INEGI, 2016; OCDE, 2016), como consecuencia, no es de extrañar que los estudiantes con CF Muy pobre sólo representen un poco más de 3\% de la población estudiada.

Pese a que el gobierno federal señala que "la población de menores ingresos en ocasiones tiene menos posibilidades de acceder a una educación de calidad y concluir sus estudios" (SEP, 2013, p. 29), vemos que, para el caso de la población estudiada, no necesariamente es así; tal parece que una declaración más coherente con los hallazgos encontrados diría que "la población de menores ingresos en ocasiones muy remotas tiene la posibilidad de acceder a una educación de calidad y concluir sus estudios".

Asimismo, se puede afianzar la conclusión de Mejía (2013), al estimar que a los estudiantes con CF Muy pobres no les fue posible reemplazar, con recursos propios, lo que la escuela no les proveyó para poder participar en un futuro próximo en la construcción de una sociedad productiva y sustentable; es decir, llegar a estudios universitarios y poder concluirlos con éxito, ya que los resultados de este estudio muestran que sólo un estudiante, de los 413 que participaron en esta investigación, cuenta con un CF Muy pobre, con un CE Alto y con una TEU Alta. Esto es, sólo se encontró a un estudiante universitario con serias desventajas sociales, económicas y culturales, que tuvo éxito antes y durante sus estudios universitarios.

Otros datos que llaman la atención, relacionados con los estudiantes denominados como Héroes y Superhéroes, se refiere a que entre ellos no existen trayectorias escolares universitarias Muy bajas, mientras que $2.4 \%$ de los Herederos presenta este tipo de trayectorias universitarias. Se puede suponer que esto se 
debe a un menor compromiso escolar los Herederos, debido a que algunos de estos estudiantes no vean en sus estudios universitarios un acceso a una mejor calidad de vida, mientras que, para los Héroes y Superhéroes, los estudios universitarios sí representan esa posibilidad. No obstante, se necesitan más estudios para explicar estos hallazgos.

Por otra parte, esta investigación también muestra resultados alentadores, ya que alrededor de $82 \%$ de los estudiantes tienen TEU entre Altas y Regulares, lo que se aproxima a la declaración del gobierno federal al señalar que "cada estudiante debe lograr un sólido dominio de las disciplinas y valores que deben caracterizar a las distintas profesiones" (SEP, 2013, p. 27). No obstante, $50 \%$ de esas TEU pertenecen a los estudiantes denominados como Herederos, quienes tienen una representatividad de 58.1\% en la población estudiada. Por ello, al igual que los hallazgos de Casillas et al. (2007), los resultados de este estudio también nos llevan a considerar que el CC que posea un individuo está relacionado con el éxito que logra en sus estudios profesionales.

$\mathrm{Al}$ respecto, pese a que todos los estudiantes de diferentes niveles socioeconómicos pueden participar en el proceso de selección y a que, efectivamente, sea común que exista en universidades públicas una heterogeneidad de estudiantes, Casillas et al. (2007) afirman que la educación superior es excluyente, pues es habitual que ingresen, permanezcan y egresen de estudios universitarios los estudiantes socioeconómicamente más favorecidos, los Herederos. También debemos considerar que el sistema pone a competir a los estudiantes más necesitados con los más privilegiados en un mismo contexto educativo, por lo que, según los hallazgos encontrados, este estudio coincide con Casillas et al. (2007), al observar que, en realidad, para el caso de la población estudiada, la educación superior es un bien al cual acceden y tienen éxito los grupos sociales más favorecidos, los que, entre la población en general, representan una minoría (AMAI, 2016).

De esta forma es evidente, como concluyen Araiza-Lozano y Audelo (2017), que algunos estudiantes, provenientes de contextos socioeconómicamente menos favorecidos, hacen y han hecho grandes esfuerzos para ingresar y permanecer en la universidad, pues existen desventajas que los estudiantes socioeconómicamen- 
te menos favorecidos tienen en comparación de sus pares que provienen de familias con mejores recursos socioeconómicos y culturales.

Por lo tanto, los resultados de esta investigación, al igual que los de Casillas, de Garay, Vergara y Puebla (2001); Chain y Jácome (2007); de Garay (2002); Guzmán y Serrano (2007), concuerdan en que no todos los estudiantes cuentan con condiciones favorables para continuar con sus estudios profesionales, ya que entre ellos existen diferencias socioculturales. En otras palabras, existe una población socioculturalmente heterogénea entre los estudiantes universitarios, la cual debe identificarse claramente para atender con la mayor eficiencia posible a cada grupo de estudiantes de acuerdo con sus necesidades. Por último, este estudio puede ser replicado en cualquier institución universitaria, se invita a los profesionales relacionados con la educación superior a que hagan réplicas de este trabajo e incluso ampliaciones del mismo, con el fin de cotejar los avances en equidad educativa que 194 la educación superior requiere.

\section{REFERENCIAS BIBLIOGRÁFICAS}

AMAI. (2016). Niveles Socioeconómicos AMAI. Recuperado de http://nse.amai.org/nseamai2/

ANUIES. (2000). La educación superior en el siglo XXI: lineas estratégicas de desarrollo: una propuesta de la ANUIES. México: ANUIES. Recuperado de http://planeacion.uaemex.mx/ InfBasCon/LaEducacionSuperiorenelSigloXXI.pdf

Araiza-Lozano, M. y Audelo López, C. (2017). El origen social de los estudiantes universitarios y su trayectoria escolar universitaria. En XIV Congreso Nacional de Investigación Educativa. Ponencia llevada a cabo en el congreso COMIE. Arzate, J. y González, J. (2007). Diversificación, crecimiento y desigualdad en la educación superior: la dimensión relativa de la universidad pública en México. Tiempo de Educar, 8(16), 277-303.

Blanco, Á. (2010). Creencias de Autoeficacia de Estudiantes Universitarios: Un Estudio Empírico Sobre La Especificidad del Constructo. RELIEVE, Revista Electrónica de Investiga- 
ción y Evaluación Educativa, 16(1), 1-28. Recuperado de http://www.uv.es/RELIEVE/v16n1/RELIEVEv16n1_2.htm

Bourdieu, P. (1984). Los tres estados del capital cultural. Sociológica, 5, 11-17. http://doi.org/10.1215/00182168-81-3-4-823

Bourdieu, P. (2005). Capital cultural, escuela y espacio social. México: Siglo XXI.

Bourdieu, P., y Passeron, J. (2009). Los Herederos. Los estudiantes y la cultura. Argentina: Siglo XXI.

Camposeco, F. (2012). La autoeficacia como variable en la motivación intrinseca y extrinseca en matemáticas a través de un criterio étnico. UCM. Universidad Complutense de Madrid. Recuperado de http://eprints.sim.ucm.es/16670/1/T34002.pdf

Casillas, M. A., de Garay, A., Vergara, J. y Puebla, M. (2001). Los estudiantes de la UAMA, un sujeto social complejo. Revista Mexicana de Investigación Educativa, 6, 139-163.

Casillas, M., Chain, R., y Jácome, N. (2007). Origen social de los estudiantes y trayectorias estudiantiles en la Universidad Veracruzana. Revista de La Educación Superior, XXXVI (2) (142), 7-29. Recuperado de http://doi.org/ISSN 0185-2760

CENEVAL. (2018). Centro Nacional de Evaluación para la Educación Superior (CENEVAL). Recuperado de http://www. ceneval.edu.mx/ceneval-web/content.do?page $=0$

Chaín, R. (1994). Demanda, selección y primer ingreso en la Universidad Veracruzana, generación 1993-1994. Xalapa, Veracruz, México: Universidad Veracruzana.

Chaín, R., y Jácome, N. (2007). Perfil de ingreso y trayectoria escolar en la Universidad. Veracruz: Artefacto Ediciones.

Conafe. (2018). Lider para la educación comunitaria. Recuperado de https://www.gob.mx/conafe

CONEVAL. (2017a). CONEVAL informa la evolución de la pobreza 2010-2016. México: CONEVAL.

CONEVAL. (2017b). Indicadores de carencia social. Recuperado de http://www.coneval.org.mx/Medicion/Paginas/Medición/Indicadores-de-carencia-social.aspx

Countrymeters. (2017). Población de México. Recuperado de http://countrymeters.info/es/Mexico

Cu Balán, G. (2005). El impacto de la escuela de procedencia del nivel medio superior en el desempeño de los alumnos 
en el nivel universitario. Revista Electrónica Iberoamericana Sobre Calidad, Eficacia y Cambio en Educación, 3(1), 764-769. Recuperado de http://www.redalyc.org/articulo.oa?id=55130171

De Garay, A. (2002). Los actores desconocidos. Una aproximación al conocimiento de los estudiantes. Sociológica, 17(49), 361-366.

De Garay, A. (2005). En el camino de la universidad. Las diversas formas de transitar de los alumnos en el primer año de la licenciatura. Colima: Universidad de Colima. Recuperado de http://www.fundacionucol.org/wp-content/ uploads/2013/09/En-el-camino-de-la-universidad.pdf

Garbanzo, G. (2013). Factores asociados al rendimiento académico en estudiantes universitarios desde el nivel socioeconómico: Un estudio en la Universidad de Costa Rica. Revista Electrónica Educare, (17). Recuperado de http:// www.scielo.sa.cr/pdf/ree/v17n3/a04v17n3.pdf

196 García-Castro, G., y Bartolucci, J. (2007). Aspiraciones educativas y logro académico. Un estudio de caso sobre características y condiciones sociales de los estudiantes de la UAM. Revista Mexicana de Investigación Educativa, 12, 1267-1288.

Gómez, E., y Kotliarenco, M. A. (2010). Resiliencia Familiar: un enfoque de investigación e intervención con familias multiproblemáticas. Revista de Psicología, 19, 103-131.

Guzmán, C. (2004). Entre el estudio y el trabajo. Revista de La Educación Superior, XXXIV(135), 155-161.

Guzmán, C. (2011). Avances y retos en el conocimiento sobre los estudiantes mexicanos de educación superior en la primera década del siglo XXI. Perfiles Educativos, 33(SPE), 91-101. Recuperado de http://www.scielo.org.mx/scielo. php? script=sci_arttext $\&$ pid $=$ S0185-26982011000500009\&ln $\mathrm{g}=\mathrm{es} \& \mathrm{nrm}=\mathrm{iso} \& \mathrm{t} \operatorname{lng}=\mathrm{es}$

Guzmán, C. (2012). Quedar afuera: experiencias y vivencias de los jóvenes que no logran ingresar a la universidad. Cultura y Representaciones Sociales, 6(12), 131-164.

Guzmán, C., y Saucedo, C. (2005). La investigación sobre alumnos en México: recuentos de una década (1992-2002). Sujetos, actores y procesos de formación. México: COMIE. 
Recuperado de http://www.comie.org.mx/doc/portal/ publicaciones/ec2002/ec2002_v12_t1.pdf

Guzmán, C., y Serrano, O. (2007). Los cambios en la composición social de la población estudiantil de la UNAM (19852003). En Estudiantes universitarios: cinco acercamientos, (pp. 164-208). México: IISUE.

Hernández, C. (2016). Diagnóstico del rendimiento académico de estudiantes de una escuela de educación superior en México. Revista Complutense de Educación, 27, 1369-1388.

IBM. (2011). SPSS Statistics for Windows. Armonk, Nueva York: IBM Corp.

INEE. (2004). Contexto sociodemográfico. La población total y en edad escolar y su crecimiento. México: INEE.

INEE. (2007). Infraestructura escolar en las primarias y secundarias de México. México: INEE.

INEGI. (2011). Sistema nacional de clasificación de ocupaciones 2011 SINCO. Aguascalientes: INEGI.

INEGI. (2016). Encuesta Nacional de los Hogares (ENH) 2016. México: INEGI. Recuperado de http://www.beta.inegi. org.mx/proyectos/enchogares/regulares/enh/2016/default.html

Jadue, G. (1996). Características familiares de los hogares pobres que contribuyen al bajo rendimiento o al fracaso escolar de los niños 1. Revista de Psicología de La PUCP, XIV(1), 35-45.

Mejía, F. (2013). Resultados del sistema educativo mexicano en su contexto ¿Coartada estructural o estructura que coarta? Revista Latinoamericana de Estudios Educativos, XLIII(4), 55-65.

Microsoft Corporation. (2016). Excel. Santa Rosa, California: Microsoft Corporation.

Miller, D. (2012). Equidad educativa versus desigualdad social: El caso del Programa Nacional de Becas en Educación Superior (PRONABES) en México. Universidades, LXII, 21-28. Recuperado de http://www.redalyc.org/articulo. oa? $\mathrm{id}=37331243003$

OCDE. (2016). Resultados Clave Pisa 2015. París: OCDE. Recuperado de http://doi.org/http://dx.doi.org/10.1787/9789264266490-en

OCDE. (2018). Estadisticas. París: OCDE. Recuperado de http:// www.oecd.org/centrodemexico/estadisticas/ 
Ordorika, I., y Rodríguez, R. (2012). Cobertura y estructura del Sistema Educativo Mexicano: problemática y propuestas. En Plan de Diez Años Para Desarrollar El Sistema Educativo Nacional, (pp. 197-222), México: UNAM.

Oros, L. (2009). El valor adaptativo de las emociones positivas. Una mirada al funcionamiento psicológico de los niños pobres. Interamerican Journal of Psychology, 43, 288-296.

Pérez-Sánchez, A., y Castejón, J. (2009). Un modelo causal-explicativo sobre la influencia de las variables psicosociales en el rendimiento académico. Bordón, 50(2), 171-185.

Rodríguez, F., Palma, X., Romo, A., Escobar, D., Aragú, B., Espinoza, L., Gálvez, J. (2013). Hábitos alimentarios, actividad física y nivel socioeconómico en estudiantes universitarios de Chile. Nutrición Hospitalaria, 28(2), 447-55. http://doi.org/10.3305/nh.2013.28.2.6230

Ruiz, N. (2012). La definición y medición de la vulnerabilidad social. Un enfoque normativo. Investigaciones Geográficas,

(77). Recuperado de http://www.redalyc.org/articulo. oa?id $=56923353006$

SEP. (2001). Programa Nacional de Educación 2001-2006. México: SEP. Recuperado de http://www.google.com.mx/url?s $a=t \& r c t=j \& q=\& e s r c=s \&$ source $=w e b \& c d=1 \& v e d=0 C C g Q$ FjAA\&url=http://planipolis.iiep.unesco.org/upload/Mexico/Mexico Programa nacional de educacion 2001-2006. pdf\&ei=1uVfU-_hGOSu2gWx1oHQDg\&usg=AFQjCNG76

SEP. (2007). Programa Sectorial de Educación 2007-2012. México: SEP.

SEP. (2013). Programa Sectorial de Educación 2013-2018. México: SEP. Recuperado de http://planipolis.iiep.unesco.org/ upload/Mexico/Mexico_Programa_sectorial_de_educacion_2013-2018.pdf

Silas, J. C. (2008). ¿Por qué Miriam sí va a la escuela? Resiliencia en la educación básica mexicana. Revista Mexicana de Investigación Educativa, 13, 1255-1279. Recuperado de http://udg.redalyc.org/articulo.oa?id=14003910

Silva, M. (2011). El primer año universitario un tramo crítico para el éxito académico. Perfiles Educativos, 33, 102-114. 